\title{
Influence of Thermal Turbulence in a Convective Ascending Stream on Phase Fluctuations of a Laser Beam
}

\author{
M. Bertolotti, M. Carnevale, B. Crosignani, and P. Di Porto
}

\begin{abstract}
The effects of thermal turbulence on the phase fluctuations of a laser beam are investigated in laboratory. The turbulent region created by means of a horizontal heated Nichrome grid is made to shift upwards owing to the convective motion. A Mach-Zehnder interference experiment is performed in which two beams from a laser source are superimposed after crossing the turbulent region. The displacements of the fringe pattern allow one to study the temporal decay of the mean square refractive index fluctuation. An interpretation of the results is given on the basis of the theory of an isotropic turbulent scalar field.
\end{abstract}

\section{Introduction}

The effects of atmospheric turbulence on the propagation of a laser beam under near earth conditions have been experimentally investigated by many authors. In particular, a certain amount of work has been recently devoted to the study of the phase fluctuations a laser beam undergoes when crossing turbulent atmosphere. ${ }^{1,2}$ The experimental results are in general contrasted with the classical Tatarski's theory of propagation in a turbulent medium. ${ }^{3}$

As is well known, phase fluctuations of a laser beam propagating in atmosphere are affected by refractive index variations, which are mainly due to temperature random changes, the phenomenon usually termed as thermal turbulence. ${ }^{4}$ This fact leads one to consider, as a very rough model for studying near earth propagation, the case in which turbulence is generated by the heating of the ground and then made to upstream by the convective motion. The relevance of such a model to real environmental conditions is strongly limited by the presence of wind, the effects of which cannot in general be neglected. We have reproduced in the laboratory the extreme case in which this effect is not present in order to obtain a first approximation scheme for studying horizontal propagation of a laser beam above ground level.

The turbulent field has been artificially generated by using a grid of heated Nichrome wires placed in a horizontal position. Its influence on the phase fluctuations

M. Bertolotti is with the Istituto di Fisica della Facoltà di Ingegneria, Università di Roma, Città Universitaria, P. Le Scienze 5, Roma, Italy. The other authors are with Fondazione U. Bordoni, Istituto Superiore P. T., Viale Trestevere 189, Roma, Italy.

Received 13 November 1968. of a laser beam propagating at various distances from the grid has been tested under different experimental conditions. A Mach-Zehnder interference experiment has been performed in which the two interfering beams from the laser source have been made to cross the turbulent zone at different distances $x$ from the heated grid. The displacements of the fringe pattern obtained in this way furnish direct information on the mean square value of the phase fluctuations $\left\langle(\Delta \phi)^{2}\right\rangle$. The behavior of this quantity has been studied as a function of $x$, of the distance $d$ between the interfering beams, and of the grid temperature. The experimental results are found to be in good agreement with a simple theoretical model.

\section{Theoretical Analysis}

The assumptions basic to our model are that the correlation function of the density fluctuations of the turbulent medium obeys a diffusion process, the convective motion playing only the role of shifting the turbulent field upwards. In other words, the mean square refractive index in a given zone only depends on the time spent by the perturbation to travel the distance from the grid to the considered zone. This equivalence between spatial and temporal decays makes our model similar to the one recently described for an analogous experiment. ${ }^{5}$ The reader is, therefore, referred to Ref. 5 for the details of the calculations.

We wish to recall the theoretical expression of the mean square value $\left\langle(\Delta \phi)^{2}\right\rangle$ of the phase fluctuations obtained by assuming a geometry of the problem as the one sketched in Fig. 1 and a diffusionlike behavior for the refractive correlation function that describes the turbulent field generated by the grid placed in $x=0$. The final result reads

$$
\left\langle(\Delta \phi)^{2}\right\rangle=\frac{D}{t}(8 \chi t)^{\frac{1}{2}}\left[1-\exp \left(-\frac{d^{2}}{8 \chi t}\right)\right]\left\{\frac{L}{(8 \chi t)^{\frac{1}{2}}} \operatorname{erf}\left[\frac{L}{(8 \chi t)^{\frac{1}{2}}}\right]\right.
$$




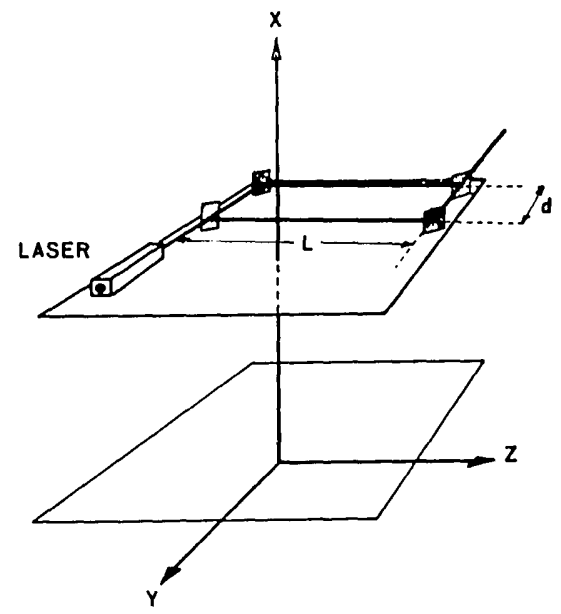

Fig. 1. Schematic geometry of the problem.

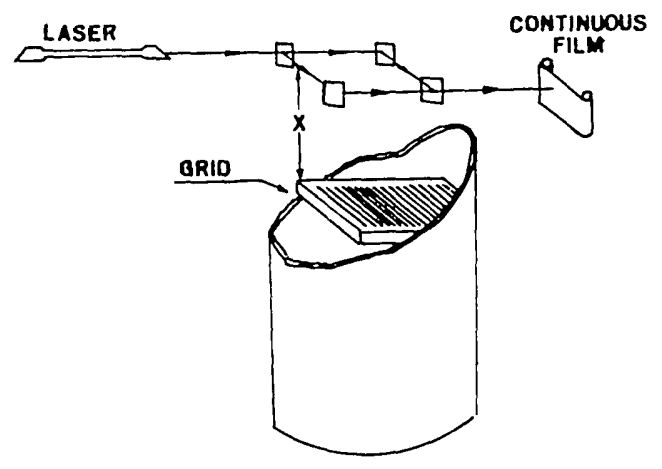

Fig. 2. Experimental setup.

$$
\left.+\frac{1}{\pi^{\frac{1}{2}}} \exp \left(\frac{-L^{2}}{8 x t}\right)-\frac{1}{\pi^{\frac{1}{2}}}\right\}
$$

with

$$
\operatorname{erf}(z)=\frac{2}{\pi^{\frac{1}{2}}} \int_{0}^{z} \exp \left(-x^{2}\right) d x
$$

where $D$ is a constant depending on the strength of turbulence, $L$ the common length of the path the two interfering beams travel in the turbulent medium, $d$ their mutual separation, and $\chi$ the heat conductivity of the air. Here $t$ is the time spent by the perturbation generated at $x=0$ to travel, owing to the convective motion, the distance $x$ to the interfering beams. We note that Eq. (1) differs from Eq. (7) of Ref. 5 by the presence of the factor $\left[1-\exp \left(-d^{2} / 8 \chi t\right)\right]$. In effect, the quantity $\exp \left(-d^{2} / 8 \chi t\right)$ is no longer negligible as it was in the conditions of the experiment reported in Ref. 5 . If $\bar{v}$ is the mean velocity of the ascending stream, which will be, in general, a certain function $\bar{v}(x)$ of the distance, we assume $t \sim x / \bar{v}$. Thus, the knowledge of $\vec{v}(x)$ allows one to express $\left\langle(\Delta \phi)^{2}\right\rangle$ as a function of $x$ alone. The explicit form of $v(x)$ can be obtained with good approximation by considering a schematic model of free convection, ${ }^{6}$ which obeys the equation:

$$
(1 / 2)(d / d x) v^{2}(x)=g \beta\left[T(x)-T_{f}\right]
$$

where $g$ is the gravity acceleration, $\beta$ the air coefficient of volume expansion, $T(x)$ the temperature at a distance $x$, and $T_{f}$ the temperature of the undisturbed fluid far removed from the grid. Equation (2) may be integrated by assuming a typical behavior of $T(x)$, for example, $T(x)=\Delta T \exp (-x / l)+T_{f}$. Here $l$ is a characteristic decay length and $\Delta T=T_{i}-T_{f}$ is the difference between the temperatures near and far from the grid. In the following, we assume

$$
\begin{aligned}
& \bar{v}(x) \simeq\left\langle v^{2}\right\rangle^{\frac{1}{2}}=\left[\frac{1}{x} \int_{0}^{x} v^{2}\left(x^{\prime}\right) d x^{\prime}\right]^{\frac{1}{2}}=(2 g \beta \Delta T)^{\frac{1}{2}} \\
& \times\left\{1-\frac{l}{x}\left[1-\exp \left(-\frac{x}{l}\right)\right]\right\}^{\frac{1}{2}} .
\end{aligned}
$$

Equation (1), which looks rather involved, actually simplifies in our experimental conditions. As a matter of fact, the ratio $L / 8 \chi t$ remains always much greater than one, so that

$$
\left\langle(\Delta \phi)^{2}\right\rangle=(D L / t)\left[1-\exp \left(-d^{2} / 8 x t\right)\right] .
$$

This expression is suitable for an experimental test on the dependence from the various parameters. They are the distance $x$ from the grid, through the relation $t=x / \bar{v}(x)$, the separation $d$ between the interfering

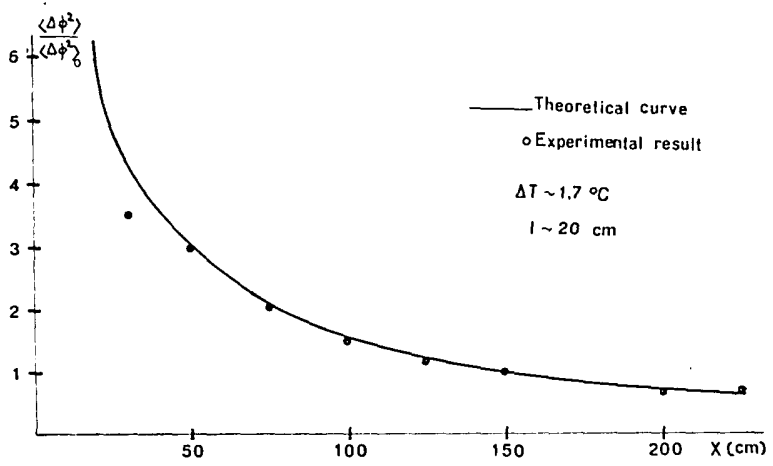

Fig. 3. Theoretical behavior and experimental values of $\left\langle(\Delta \phi)^{2}\right\rangle$ vs the grid distance $x\left(\Delta T \sim 1.7^{\circ} \mathrm{C}, l \sim 20 \mathrm{~cm}\right)$.

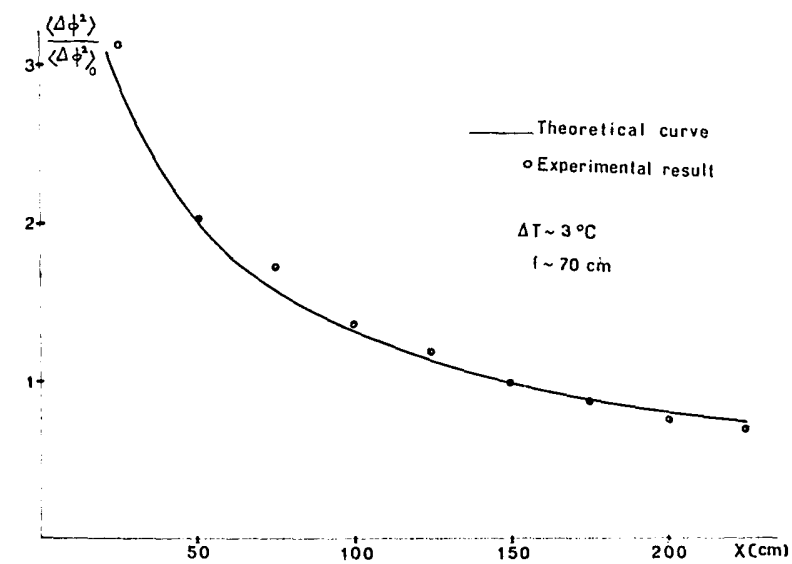

Fig. 4. Theoretical behavior and experimental values of $\left\langle(\Delta \phi)^{2}\right\rangle$ vs the grid distance $x\left(\Delta T \sim 3^{\circ} \mathrm{C}, l \sim 70 \mathrm{~cm}\right)$. 


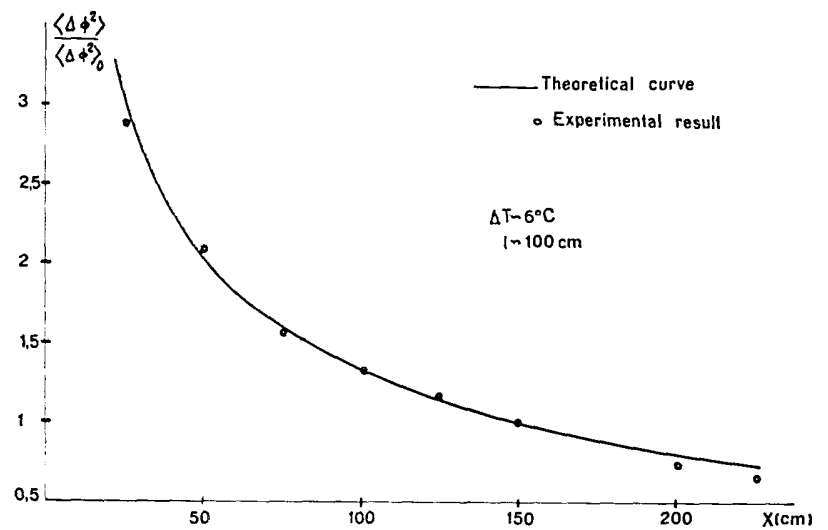

Fig. 5. Theoretical behavior and experimental values of $\left\langle(\Delta \phi)^{2}\right\rangle$ vs the grid distance $x\left(\Delta T \sim 6^{\circ} \mathrm{C}, l \sim 100 \mathrm{~cm}\right)$.

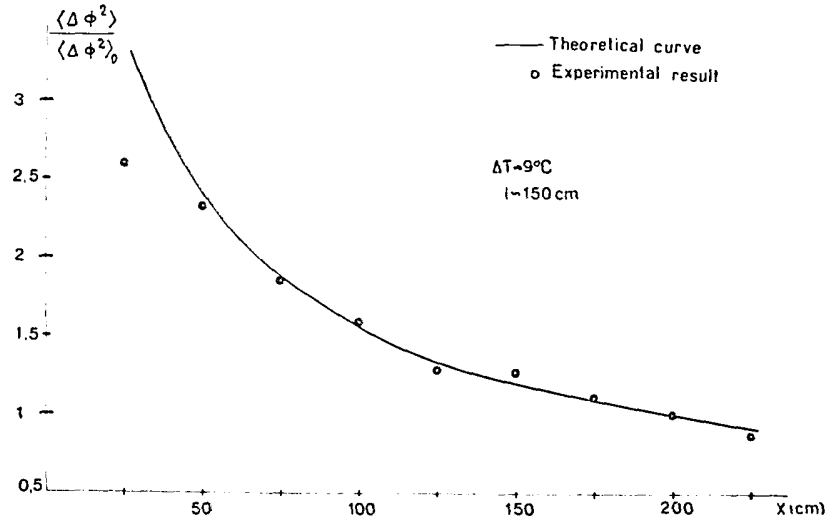

Fig. 6. Theoretical behavior and experimental values of $\left\langle(\Delta \phi)^{2}\right\rangle$ vs the grid distance $x\left(\Delta T \sim 9^{\circ} \mathrm{C}, l \sim 150 \mathrm{~cm}\right)$.

beams and the grid temperature, which influences the factor $\Delta T$ contained in the expression of $\tilde{v}$.

We wish finally to note that the theoretical expression of $\left\langle(\Delta \phi)^{2}\right\rangle$ is meaningless for small values of $x$, since the hypothesis of homogeneous isotropic turbulence, on which the theory is based, is in this case no longer valid.

\section{Description of the Experiment and Results}

The experimental setup is schematically represented in Fig. 2. The turbulent region created around the heated Nichrome grid shifts upwards following the convective motion and is shielded from external disturbances by means of a tube $\sim \frac{1}{2} \mathrm{~m}$ in diameter. A $6328-\AA \mathrm{He}-\mathrm{Ne}$ laser beam is brought to interfere through a Mach-Zehnder interferometer at various distances from the grid. The resulting interference pattern is recorded through a narrow slit by a continuously moving camera, with a speed of $6 \mathrm{~cm} / \mathrm{sec}$. The movement of the recorded fringes appears on the film as a continuously varying pattern of lines. The information on phase variations is extracted by sampling the position of the fringes at time intervals of $10^{-2}$ sec. Each run refers to measurements made at a fixed distance $x$ and lasts about 5-10 sec. The dependence of $\left\langle(\Delta \phi)^{2}\right\rangle$ vs distance, for distances ranging from $30 \mathrm{~cm}$ to $225 \mathrm{~cm}$, is then scanned for a fixed value of the grid temperature. Measurements are performed for four different values of this temperature. The distance between the interfering beams, and their path in the turbulent medium are kept constant and equal, respectively, to $7 \mathrm{~cm}$ and $27 \mathrm{~cm}$. The experimental and theoretical values of $\left\langle(\Delta \phi)^{2}\right\rangle$, normalized to a suitable point, are plotted in Figs. 3-6, each figure corresponding to a fixed $\Delta T$ and $l$. The experimental values of the parameters $\Delta T$ and $l$ have been obtained by scanning the temperature field as a function of $x$, by means of a thermocouple thermometer.

The agreement between theory and experiment is satisfactory down to distances below which the turbulent field can no longer be assumed as homogeneous. This is due to the combined effects associated with the size of the grid and the ascending velocity.

The dependence of $\left\langle(\Delta \phi)^{2}\right\rangle$ on the distance $d$ between the interfering beams has also been tested. To this end, a contemporary measurement of the phase fluctuation associated with different values of $d$ has been performed by using a multibeam interferometer. This has been done by coupling two four-beam interferometers of the kind already used for atmospheric turbulence measurements. $^{2}$

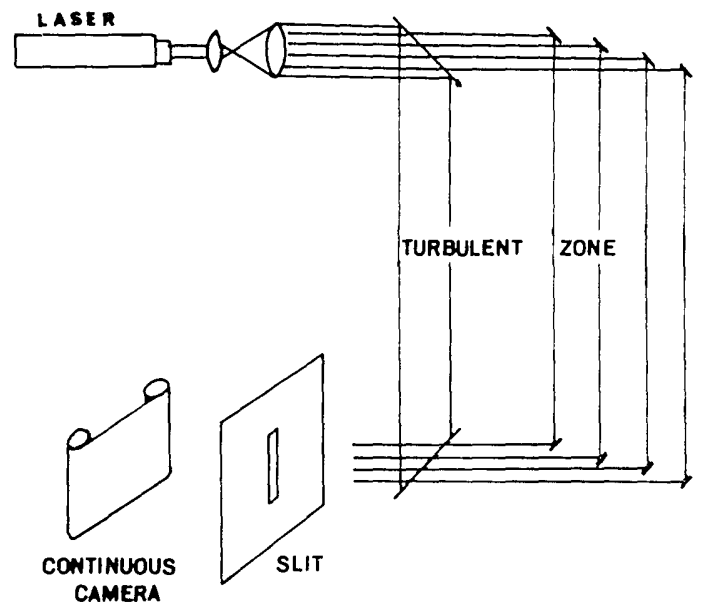

Fig. 7. Expermental geometry for testing the behavior of $\left\langle(\Delta \phi)^{2}\right\rangle$ with the beam separation.

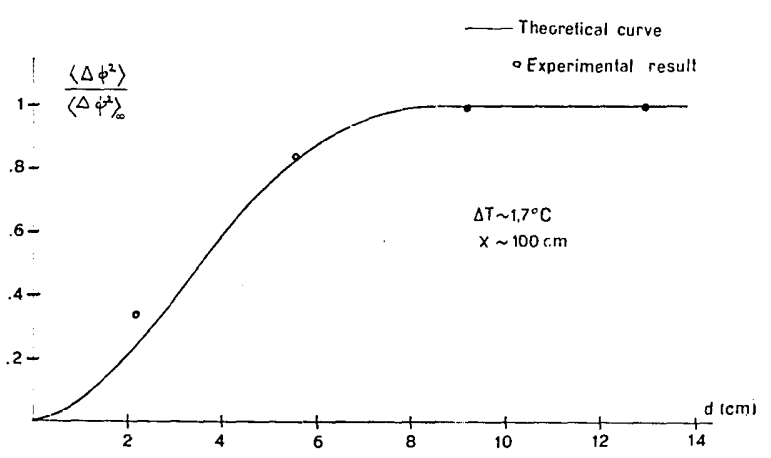

Fig. 8. Theoretical behavior and experimental values of $\left\langle(\Delta \phi)^{2}\right\rangle$ vs the beam separation $d\left(\Delta T \sim 1.7^{\circ} \mathrm{C}, x \sim 100 \mathrm{~cm}\right)$. 


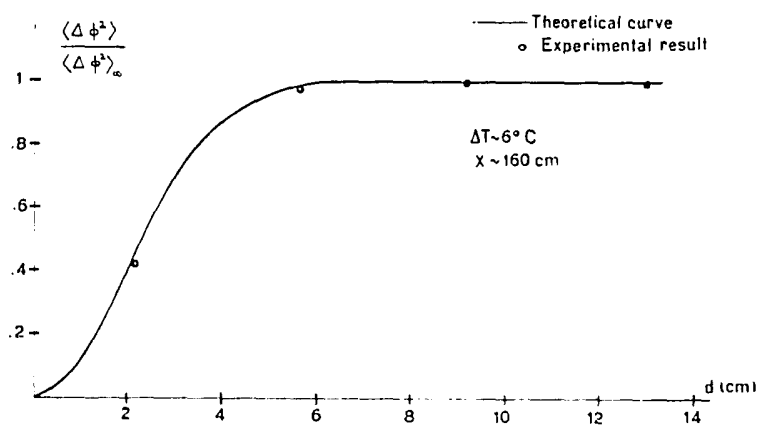

Fig. 9. Theoretical behaviors and experimental values of $\left\langle(\Delta \phi)^{2}\right\rangle$ vs the beam separation $d\left(\Delta T \sim 6^{\circ} \mathrm{C}, x \sim 160 \mathrm{~cm}\right)$.

The geometry of the experiment is schematically sketched in Fig. 7. This allows one to study the fluctuations of the four systems of fringes obtained by allowing four beams to interfere with a fixed one, the actual values of $d$ being $2.2 \mathrm{~cm}, 5.7 \mathrm{~cm}, 9.2 \mathrm{~cm}$, and $13.2 \mathrm{~cm}$. Figures 8 and 9 refer to experimental situations in which the distance from the grid is fixed, thereby obtaining constant value of $t$.

The theoretical values of the expression $1-\exp \left(-d^{2} /\right.$ $8 \chi t)$ and the corresponding experimental values $\langle[\Delta \phi$ $\left.(d)]^{2}\right\rangle /\left\langle[\Delta \phi(\infty)]^{2}\right\rangle$ are there reported as a function of $d$, for two different temperatures. The saturation behavior of $\left\langle[\Delta \phi(d)]^{2}\right\rangle$ vs distance $d$ is clearly shown.

\section{Conclusions}

We have experimentally tested a simple theoretical model, which yields a first approximation description of the influence of thermal turbulence on the phase fluctuations of a laser beam propagating under near earth conditions. More precisely, this model roughly represents outdoor situations in which the heating of the ground can be regarded as the main source of the thermal turbulent field. The experiment has been performed in the laboratory by studying the effect of a horizontal heated grid on the phase fluctuations of a laser beam propagating at various heights.
Our results are in agreement with the assumption that the turbulent field created around the grid is simply shifted by the convective ascending stream.

We wish to note the difference between this situation and the one reported in Ref. 5 . In that case, the thermal field was horizontally shifted by means of a blower, which produced a constant velocity. In the present case, conversely, the convective velocity is a function of the distance from the grid, this giving rise to significant changes. As a matter of fact, while in Ref. 5 the behavior of $\left\langle(\Delta \phi)^{2}\right\rangle$ in relation to the distance is of the type $1 / x$, in the present case this is only approximately true for intermediate value of $x$. For small and great values of $x$, the behavior is, respectively, of the type $1 / x^{\frac{3}{2}}$ and $1 / x^{2}$.

The validity of the model has been confirmed at least up to ascending velocities of the order of $1 \mathrm{~m} / \mathrm{sec}$. An important point is the fact that the model predicts a saturation behavior for $\left\langle(\Delta \phi)^{2}\right\rangle$ with increasing distance between the interfering beams similar to that effectively found in atmospheric measurements. ${ }^{2,7}$

We wish to thank $\mathrm{D}$. Sette for having pointed out the problem. The technical assistance of $\mathrm{E}$. Bezzi is gratefully acknowledged.

This work was partially supported by the Italian National Council of Research.

\section{References}

1. See, for example, P. Burlamacchi, A. Consortini, and L. Ronchi, Appl. Opt. 6, 1273 (1967).

2. M. Bertolotti, M. Carnevale, L. Muzii, and D. Sette, Appl. Opt. 7, 2246 (1968).

3. V. I. Tatarski, Wave Propagation in a Turbulent Medium (McGraw-Hill Book Company, Inc., New York, 1961).

4. H. Hodara, Proc. IEEE 54, 368 (1966).

5. M. Carnevale, B. Crosignani, and P. Di Porto, Appl. Opt. 7. 1121 (1968).

6. A. J. Chapman, Heat Transfer (The Macmillan Company, New York, 1960), p. 266.

7. P. Burlamacchi, A. Consortini, L. Ronchi, and G. Toraldo di Francia, URSI Symposium on Electromagnetic Waves, Stresa, Italy, 1968.
Meetings Calendar continued from page 1110

\section{September}

1-5 Low-Frequency Vibrational Spectroscopy, Reunion EUCHEM M. Bigorgne, CNRS, ENSC, Paris, France

2-4 APS Mtg., Hawaii W. W. Havens, Jr., 335 E. 45th St., New York, N.Y. 1001i

2-6 Molecular Structure and Spectroscopy Symp., OSU K. N. Rao, Phys. Dept., OSU, 174 W. 18th Ave., Columbus, Ohio

2-6 Identification of Small Particles course, London M. L. Fallert, McCrone Res. Inst., 451 E. 31 St., Chicago, Ill. 60616

3-10 British Assoc. for the Advancement of Sci., 131st Ann. Mtg., Exeter BAAS, 20 Great Smith St., London SW 1, England

7-12 Stellar Rotation Colloq., OSU A. Slettebak, Astron. Dept., OSU, 174 W. 18 Ave., Columbus, Ohio 43210

8-12 Laser Fundamentals and Applications course, PIB, Farmingdale PIB, 333 Jay St., Bklyn, N.Y. 11201
8-12 Inter/Micro-69, Internat. Conf. on Microscopy, London $W$. C. McCrone, McCrone Res. Insl., 451 E. 31st St., Chicago, Ill. 60616

9-12 Nonlinear Optics Conf., The Queen's U., Belfast M. H. Key, Dept. Pure and Appl. Phys., The Queen's U., Belfast 7, Northern Ireland

10-October 10 Austrian Physical Soc., Salzburg, Austria K. H. Riewe, German Phys. Soc., Herawaus St. 12-14, 645 Hanau/Main, Germany

15-19 Photomicrography course, London $M$. L. Fallert McCrone Res. Inst., 451 E. 31 St., Chicago, Ill. 60616

15-26 Spectroscopic Techniques in Organic Chemistry course, UCLA Eng. and Phys. Sci. Ext., U. of Calif., Los Angeles, Calif. 90024

16-19 3rd Ann. Solid State Devices Conf., U. of Exeter England IEEE, 345 E. 47 St., New York, N.Y. 10017

21-26 Energy Conversion Eng. Conf., Statler Hilton, Washington, D.C. J. Henry, AIChE, 335 E. 47 th St., New York, N.Y. 10017 